\title{
ANÁLISE TÉCNICA DE ESTUDOS AMBIENTAIS DA ATIVIDADE PETROLIFERA ONSHORE NO RIO GRANDE DO NORTE
}

\author{
Maria Herlaiane Nascimento Costa \\ Concluinte no Curso Técnico em Meio Ambiente - IFRN Campus Natal Central \\ E-mail: herlaiane@hotmail.com \\ Rosiney Araújo Martins \\ Professora da Diretoria de Recursos Naturais - IFRN Campus Natal Central \\ E-mail: rosiney@cefetrn.br \\ Erika Araújo da Cunha Pegado \\ Professora da Diretoria de Recursos Naturais - IFRN Campus Natal Central \\ E-mail: erika@cefetrn.br
}

\begin{abstract}
RESUMO
O licenciamento das diferentes atividades petrolíferas tem como um dos principais instrumentos de controle de impactos ambientais a exigência de estudos ambientais que devem ser elaborados em consonância com as normatizações ambientais vigentes Lei 6938/81. No Rio Grande do Norte (RN), o estudo ambiental integrante do processo de licenciamento para a perfuração de poços exploratórios é o Relatório de Controle Ambiental, cuja aprovação pelo órgão ambiental estadual, é uma das condicionantes para a liberação da Licença de Perfuração (Lpper). Análises técnicas realizadas em estudos ambientais de diferentes estados brasileiros mostram deficiências no atendimento satisfatório aos conteúdos exigidos na legislação, e muitos empreendimentos são licenciados com estudos de baixa qualidade técnica. Análises pioneiras sobre RCA's, aprovados pelo Instituto de Desenvolvimento Sustentável e Meio Ambiente do RN (IDEMA), estão sendo desenvolvidas pelo Instituto Federal de Educação Ciência e tecnologia do Rio Grande do Norte (IFRN), como um dos resultados da realização do Curso de Especialização em Licenciamento Ambiental onshore. Os resultados preliminares mostram a tendência já identificada nos demais estados e será apresentado ao longo deste estudo, que consiste de uma pesquisa documental (análise técnica) realizada em um RCA, aprovado pelo IDEMA, para a perfuração exploratória de seis poços no município de Carnaubais/RN. A análise do RCA foi fundamentada no guia de análise técnico construído a partir de Sánchez (2006) e o Termo de referência (TR) do IDEMA.
\end{abstract}

PALAVRAS-CHAVE: Perfuração, análise técnica, Relatório de Controle Ambiental. 


\section{ANÁLISE TÉCNICA DE ESTUDOS AMBIENTAIS DA ATIVIDADE PETROLIFERA ONSHORE NO RIO GRANDE DO NORTE}

\section{INTRODUÇÃO}

A atividade petrolífera vem sendo considerada como um dos vetores importantes da economia mundial, proporcionando reflexas nas mais diversas áreas da sociedade. Geradora de riqueza, a atividade de exploração de petróleo, é potencialmente poluidora, têm seus impactos minimizados com o cumprimento efetivo das normatizações ambientais vigentes, sendo uma das mais relevantes o licenciamento ambiental.

O desenvolvimento da atividade petrolífera remonta aos meados do século XIX, porém, somente na segunda metade do século XX, a questão ambiental passou a ser considerada, com a exigência da realização da Avaliação de Impacto Ambiental - AIA (1969) para empreendimentos nos Estados Unidos e, posteriormente, em outros países desenvolvidos Sánchez, 2006. No Brasil, para as atividades de pesquisa, exploração e desenvolvimento de campos de petróleo são obrigatórias a expedição de licença ambiental, a cargo de órgão públicos das esferas federal, estadual e municipal, baseadas na legislação pertinente ao tema.

$\mathrm{Na}$ atividade de exploração petrolífera onshore, o atendimento da normatização aplicada aos empreendimentos de exploração de poços exige estudos cada vez mais apurados, em consonância com a tendência protecionista vigente desde a Conferência Rio 92 que resultou em diversos documentos internacionais e na atual tendência legislativa no sentido a busca do desenvolvimento sustentável.

O licenciamento ambiental é considerado o mais importante mecanismo de controle do poder público com relação às atividades econômicas que interferem fortemente no meio ambiente.

No âmbito federal, as principais normas que tratam do tema do licenciamento ambiental e assuntos correlatos, são: o art. 225, § 1º IV, da Constituição Federal a Lei 6.938/81, a Política Nacional do Meio Ambiente e seu regulamento (o Decreto 99.274/90); e as Resoluções 001/86, 009/87 e 237/97, do Conselho Nacional do Meio Ambiente (CONAMA).

O Rio grande do Norte possuem a atividade petrolífera onshore, sendo assim a legislação estabelece exigências de estudos ambientais para o seu licenciamento, que são propostos pelo o órgão competente do estado, no caso o IDEMA, que solicita Relatório de Controle Ambiental (RCA) para a LPper que é o documento que licencia a atividade de perfuração mediante apresentação, pelo empreendedor, desde relatório.

De acordo com Sánchez (2006 cap. 15) estudos anteriores que visam a uma avaliação critica de estudos ambientais e, principalmente, apontar suas deficiências, foram publicados por pesquisadores de vários países. Muitos desses trabalhos não só apontaram deficiências como também ajudou a formular diversas recomendações que hoje integram o conjunto de boas práticas de AIA. Diante desse fato e com base na Constituição Federal vigente, na qualidade dos estudos ambientais, e na sua relevância para o meio ambiente, o existente trabalho apresenta os resultados sobre a análise técnica de um RCA para três 
poços exploratórios de petróleo, aprovado pelo IDEMA, localizados no município de Porto de Carão, é feita uma comparação da análise desse estudo com o TR do IDEMA, com as boas práticas internacionais apresentada em Sánchez (2006), e utilizar um guia para análise técnica baseado no termo de referência e em Sánchez 2006. Como já citado, o órgão ambiental estadual, o IDEMA, é o responsável por licenciar a grande maioria dos campos petrolíferos terrestres do Rio Grande do Norte.

De acordo Cotovicz Junior 2009, O número de requisições de licenciamento ambiental cresceu muito nos últimos anos, à medida que foram descobrindo a existência de grande quantidade de petróleo na bacia Potiguar (Figura 1).



Figura 1 - Número de licenças ambientais emitidas pelo IDEMA nos período de 2006 até abril de 2009. Fonte: Silva, 2009.

Este fato releva a importância da qualidade desses estudos, e das possíveis conseqüências que ausência deles pode causar, já que no Rio Grande do Norte a indústria petrolífera é a que gera arrecadação de tributos.

Este trabalho tem o intuito de não só aponta as possíveis deficiências existentes no RCA, bem como explicitar as causas e as possíveis soluções para melhoria desses estudos, sendo assim, atingir seu objetivo central, que é o desenvolvimento sustentável.

\section{METODOLOGIA}

O trabalho é fundamentado com base em pesquisa bibliografias tendo como referencial principal o livro de Sánchez 2006, o TR do IDEMA, o guia proposto de acordo com as boas praticas internacionais, e o parecer técnico realizado, na análise técnica de um RCA para seis poços exploratórios de petróleo, aprovado pelo IDEMA, localizados no município de Porto de Carão, tendo em vista que esta é uma pesquisa documental, serão apresentados fatos que são comprovados, com base em outras pesquisas recentemente ralizadas.

\section{ANÁLISE DE UM RELÁTORIO DE CONTROLE AMBIENTAL}

As primeiras perfurações no Brasil datam entre 1892 e 1897, na localidade de Bofete-SP, porém, somente em 1981 a Lei Federal 6.938, que trata da Política nacional do Meio 
Ambiente foi promulgada. No Rio Grande do Norte as primeiras perfurações ocorreram nos anos 70 e, em 1982. A Constituição Federal (CF) explicita que qualquer pessoa pode analisar um estudo ambiental, desde que siga a legislação vigente. De acordo com CF, o meio ambiente ecologicamente equilibrado é direito de todos (CF, art. 225 caput), sendo assim o respeito ao meio ambiente e o comprimento da lei se faz necessário através de pesquisa técnica - cientifica, para averiguação da veracidade dos estudos ambientais analisados e licenciados, a importância é tanta que no cap. 15 Sánchez (2006) afirma junto com diversos autores como Teixeira et all( 1994 apud SÁNCHEZ, 2006. Pg. 389) que os estudos ambientais possuem uma baixa qualidade, em relação ao que a legislação impõem, onde também relata que a analise técnica de um estudo de impacto ambiental não é de interesse exclusivo do agente decisório, mas sim de todos os protagonistas que podem analisar os estudos e tentar influenciar o processo decisório.

Agra Filho (1993, apud SÁNCHEZ, 2006. Pg. 389) analisou vinte EIA/RIMAS, onde o autor concluiu que a ausência ou a debilidade de TR é fator que compromete todo o processo de AIA, a começar pela qualidade dos estudos apresentados. Autores como Bisset (1954b, apud SÁNCHEZ, 2006. Pg. 392), Buckley (1991a, 1991b, apud SÁNCHEZ, 2006. Pg. 392), Culhane (1987, apud SÁNCHEZ, 2006. Pg. 392) e Culhane et al (1987, apud SÁNCHEZ, 2006. Pg. 392), analisaram respectivamente, casos da Grã-Bretanha, da Austrália e dos Estados Unidos, chegaram a duas conclusões, a primeira que muitas das previsões apresentadas nesses estudos não eram passiveis de verificação, seja por não serem quantitativas, seja por diversa deficiências relacionadas à forma como eram apresentadas, como falta de indicação da abrangência espacial dos impactos ou falta de escolha da indicadores apropriados para o monitorar os impactos reais. No Brasil estudos foram conduzidos sob essa óptica, com conclusões similares (Dias e Sánchez, 2001; Prado Filho e Souza, 2004).

De acordo com Sánchez (2006) muitos projetos realmente implantados eram bastante diferentes daqueles que haviam sido descritos nos estudos de impacto ambiental, uma situação que, evidentemente dificulta ou mesmo impede qualquer comparação entre impactos previstos e reais, diante da situação atual dos órgãos ambientais brasileiros, como, alias, a maioria de seus congêneres em outros países, não fazem uma analise ou uma classificação sistemática da qualidade dos estudos apresentados, de forma tal que seja possível alguma comparação ou aferição de usa qualidade.

Sánchez (2006) relata que os órgãos ambientais que executam as diretrizes das normas estaduais têm diferentes estruturas de acesso de informações pertinentes ao processo de licenciamento ambiental, que variam de acordo com a política daquela unidade federativa. Ainda sugere como deveria ser um estudo ambiental com um balanço adequado entre descrição e análise, rigor metodológico e isenção para que se possa obter qualidade de todo estudo ambiental, tendo como objetivo da analise técnica dos estudos ambientais a busca para determinar se o estudo de impacto tem forma e conteúdo satisfatórios e adequados, pois se houver preparação previa de termos de referencias, então a analise dos estudos deve obrigatoriamente tê-los como base, mas não precisa limitar-se a eles.

O conteúdo mínimo dos estudos de impactos ambiental é determinado pela resolução CONAMA 01/86, mas os órgãos licenciadores podem ter seus próprios critérios, desde que estes não contradigam, ou seja, menos restritos que aqueles estabelecidos na norma federal, as decisões eventualmente tomadas com base em tal estudo podem ser questionadas juridicamente e consideradas nulas. 
A análise do RCA de Porto de Carão foi realizada com base no TR, o modelo a ser seguindo foi o mesmo em relação ao que os técnicos analisaram, ou seja, a mesma metodologia, em um estudo Oliveira (2009), realizou uma pesquisa que teve enfoque na visão dos técnicos, esta obteve como resultado, a má estruturação do IDEMA, com poucos técnicos, e estrutura física, além dos técnicos ter relatado a falta de uma legislação direcionada para o licenciamento onshore, pois já existe para a offshore, diante desses aspectos ainda termos o RCA como o único tipo de estudo que é solicitado para o licenciamento de perfuração e produção, sabendo, porém que na teoria, existem vários outros estudos que deveriam ser realizados, como a licença de instalação, de operação entre outros.

Cotovicz Junior (2009), analisou quatro RCAs, todos para LPper, no qual se direcionou para o atendimento do termo de referencia em relação a água, e em todos os itens analisados, 6, 7, 8 e 9, os RCAs não cumpriram por completo as exigências explicitadas no TR (anexos 1, 2, 3, 4 e 5). Em outras palavras, o termo de referência exigiu certos detalhamentos e refinamentos não verificados nos relatórios aprovados, comprometendo assim a qualidade dos estudos.

Com outra concepção Oliveira 2009, constata que com relação ao atendimento do Termo de Referência - TR dos EAs, os técnicos afirmaram que os estudos têm atendido de forma satisfatória aos TRs, divergindo com do que afirmou Cotovicz Junior (2009).

O fato é que os estudos estão de má qualidade, diante disso se cria um impasse, tendo vista, porém, que a atividade petrolífera é poluidora, sendo necessários estudos ambientais eficazes, que se possa tornar uma ferramenta de controle ambiental, para atingir o desenvolvimento sustentável, já que o Rio Grande do Norte é o maior produtor de Petróleo onshore.

Com base nesses estudos e pesquisa foi elaborado um guia de acordo com o Termo de Referência do IDEMA, e com o guia para análise técnica de estudos de impacto ambiental Sánchez (2006), onde em todos os itens foram elaboradas perguntas, de forma que possa ser mais bem interpretado na visão dos técnicos, para possível análise de RCAs.

Com este guia foi analisado um RCA de Porto do Carão, Carnaubais - RN, com intuito de se verificar as informações citadas, a cada item foi dado um laudo, como se fosse um técnico a analisar o estudo, infelizmente o resultado foi alarmante, pois se verificaram, vários itens mal atendidos, com informações básicas, com ausência de detalhamento como é solicitado no TR.

Diante de todos os aspectos levantados, o fato incontestável é que está havendo falhas, nos estudos ambientais, e estas é constatada na análise realizada, de Porto de Carão, onde são discutidos os aspectos da avaliação técnica.

\section{RESULTADOS}

Com a análise realizada de um RCA, a qual já foi citada, foram encontradas diversas falhas. Este parecer técnico apresenta uma análise do Relatório de Impactos Ambiental RCA, analisado de acordo com o guia proposto e próprio TR do IDEMA, foi feito para ajudar a identificar as deficiências, e fornecer mais apoio para a qualidade das análises 
ambientais feitas pelo IDEMA, este guia foi realizado também de acordo com o livro de Sánchez (2006).

Tabela 1- Guia proposto

\begin{tabular}{|l|l|}
\hline ITEN AVALIADO & QUESTIONAMENTO \\
\hline $\begin{array}{l}\text { 1. IDENTIFICAÇÃO DO } \\
\text { EMPREENDENDOR E DO }\end{array}$ & A informação apresentada é completa? \\
\hline $\begin{array}{l}\text { 2. IDENTIFICAÇÃO DA EMPRESA } \\
\text { RESPONSAVEL PELO RCA }\end{array}$ & A informação apresentada é completa? \\
\hline $\begin{array}{l}\text { 3.DESCRIÇÃO DO } \\
\text { EMPREENDIMENTO }\end{array}$ & Objetivos são claramente expostos? \\
\hline 4 ÁREA DE INFLUÊNCIA & $\begin{array}{l}\text { Há descrição da área de influencia direta e } \\
\text { indireta do empreendimento, para fases de } \\
\text { implantação e operação nos meios físico, } \\
\text { biológico e antrópico? }\end{array}$ \\
\hline 5 DIAGNOSTICO AMBIENTAL & $\begin{array}{l}\text { Recursos hídricos de utilização real ou } \\
\text { potencial, como mananciais, foram } \\
\text { identificados e descritos? }\end{array}$ \\
\hline $\begin{array}{l}\text { 6. IDENTIFICAÇÃO E ANÁLISE DOS } \\
\text { IMPACTOS }\end{array}$ & $\begin{array}{l}\text { Foi estabelecida com base nas fases de } \\
\text { implantação e operação do empreendimento, } \\
\text { considerando os impactos sobre os meios } \\
\text { físico, biológico e antrópico? }\end{array}$ \\
\hline 7. MEDIDAS MITIGADORAS & $\begin{array}{l}\text { São propostas medidas para todos os impactos } \\
\text { relevantes, diretos e indiretos? }\end{array}$ \\
\hline $\begin{array}{l}\text { 8 PROGRAMA DE } \\
\text { ACOMPNHAMENTO E } \\
\text { MONITORAMENTO DOS IMPACTOS } \\
\text { AMBIENTAIS }\end{array}$ & $\begin{array}{l}\text { Os principais impactos são contemplados no } \\
\text { plano, considerando-se as fases de implantação, } \\
\text { operação e desativação? }\end{array}$ \\
\hline 9. CONCLUSÕES & $\begin{array}{l}\text { Há esclarecimento do aspecto ambiental e da } \\
\text { viabilidade ou não do empreendimento? }\end{array}$ \\
\hline
\end{tabular}

Foi analisado cada item individualmente, de acordo com o TR e com o guia proposto. No item processo operacional foi constatado a falta de informação na identificação das fases de perfuração, instalação e operação, como é determinado no TR. Já no item Funcionamento da linha Surgência falta esclarecimento sobre o local e o espaço a ser adotado.

Na Área de influencia não existe descrição da área de influencia indireta, o que deixa insatisfeito esse item. No item Meio biótico falta esclarecimento sobre as estações coletoras de amostras, de forma que haja sua identificação e seu mapeamento essa importância se dar para acompanhamento desses impactos, ou seja, pois é necessário este mapeamento para o futuro acompanhamento dos impactos.

Nas Medidas mitigadoras, foi constatado que falta ser relatada a previsão de relatórios que comprovem o atendimento a esse critério, diante da realidade não são previstos estes relatórios, e é necessário, já que a fiscalização deixa a desejar. 
No item Programa de acompanhamento e monitoramento dos impactos ambientais, foi o mais afetado com a ausência de informações, dos principais impactos no plano, considerando-se as fases de implantação, operação e desativação, não descreveram as freqüências de amostragens, muito menos, a indicação e justificativa da rede de amostragem de seus métodos, considerando do mesmo assim, a responsabilidade de relatar a evolução dos impactos ambientais causados pelo empreendimento.

De acordo com todos os itens relatados e analisados, a falta de informação é constante, de esclarecimento, isso é prejudicial para o meio ambiente, pois são informações importantes que futuramente farão diferenças, para relatórios de impacto ambiental, pois o os aspectos ambientais estão sendo deixados de ser relatado, o banco de dados, está inoperante, e os prejuízos ambientais que passam está acontecendo, não estão sendo relatados pelos empreendedores, e o IDEMA, não fiscaliza. É importante ressaltar que nem o TR de referencia está sendo comprido, o que nos cerca de bastantes dúvidas, quanto a real situação desses estudos com a realidade.

\section{CONCLUSÕES}

A má qualidade dos estudos ambientais compromete todo um ecossistema, no qual fazemos parte, diante da importância que a indústria petrolífera tem para o nosso estado economicamente, considerando também, que este mercado representa mais $50 \%$ das licenças emitidas pelo IDEMA, foi feito este com intuito de melhorar os estudos ambientais, apresentando assim, as causas e realidade, dos impactos reais e coexistentes, quando todos se detêm apenas ao conhecimento econômico.

Foi verificado a carência de legislação quanto ao requisito da água, a má estruturação do IDEMA, junto com poucos profissionais e falta de treinamento especifico, a ênfase dada a este trabalho se dar, pelo fato de que o aspecto mais importante do licenciamento não está sendo cumprido, que é a parte de minimização, mitigação e acompanhamento dos impactos ambientais, o que deixa claro que este resultado indica estudos carentes, incompletos, que não segue o que na teoria impõem como a legislação e o TR.

Vale ressaltar que não é apenas um erro, uma falha, que provoca a má qualidade dos estudos, e sim, todo o procedimento para se gerar a licença, desde o não o comprimento do TR pela empresa que faz o estudo, a aceitação pelos os Técnicos do IDEMA do estudo esta forma, e que reconhecem também a falta de uma legislação direcionada para este setor, contudo falta um pouco de tudo, falta profissionais qualificados no IDEMA e nas empresas que fazem o estudo e falta uma legislação.

Mesmo diante desses problemas, é possível que estas deficiências sejam resolvidas, pois este é o objetivo, não apenas apontá-las e relatar o que está certo e errado e onde está o problema, mas mostrar, que é possível melhorar estes estudos, para que se possa conseguir um desenvolvimento sustentável para o Rio Grande do Norte. 


\section{REFERÊNCIAS BIBLIOGRÁFICAS}

AOQUI, Milton Issashi. Avaliação de Impactos ambientais - Módulo I. Natal,CEFET, 2008.

BRASIL.Constituição federativa do Brasil. lei ${ }^{\circ}$ 6.938, 31 de agosto de 1981. Dispõe sobre a Política Nacional do Meio Ambiente, seus fins e mecanismos de formulação e aplicação, e dá outras providências. Disponível em:<http://www.planalto.gov.br/ccivil_03/Leis/L6938.htm>.Acesso em: 31 de Jul. 2009.

BRASIL. Conselho nacional do meio ambiente. Resolução $n^{0}$ 237, 19 de dezembro de 1997. Regulamenta os aspectos do licenciamento estabelecido na política nacional do meio ambiente.

Disponível

em: $<$ http://www.mma.gov.br/port/conama/legiabre.cfm?codlegi=237>. Acesso em: 15 de Jul. 2009.

Conselho nacional do meio ambiente. Resolução nº 023, 07 de dezembro de 1994. Institui procedimentos específicos para o licenciamento das atividade relacionadas à exploração e lava de jazidas de combustíveis líquidos e gás natural. Disponível em:< http://www.mma.gov.br/port/ conama/legiabre.cfm?codlegi=164>. Acesso em: 16 de Jul. 2009.

Conselho nacional do meio ambiente. Resolução nº 001,23 de janeiro de 1986. Dispõe sobre critérios básicos e diretrizes gerais para a avaliação de impacto ambiental. Disponível em: $<$ http://

<www.mma.gov.br/port/conama/res/res86/res0186.html>.Acesso em: 16 de Jul. 2009.

CONSELHO ESTADUAL DO MEIO AMBIENTE DO RIO GRANDE DO NORTE. lei complementar $\mathrm{n}^{0}$ 272, de 03 de março de 2004. Disponível em: $<$ http://www.idema.rn.gov.br/contentproducao/aplicacao/idema/legislacao_ambiental/envia dos/legislacao.asp>. Acesso em: 21 de Jul. 2009

CONSELHO ESTADUAL DO MEIO AMBIENTE DO RIO GRANDE DO NORTE. Instruções Técnicas para Apresentação de Projetos. Disponível em: $<$ http://www.idema.rn.gov.br/arquivos/Licenciamento/Documentos\%20Técnicos\%20por\% 20Atividade\%20ou\%20Tipo\%20de\%20Serviço/geral_licencas/IT\%20\%20Geral\%20LP\%20ou\%20LSP.pdf. Acesso em: 30 de Jul. 2009.

CONSELHO ESTADUAL DO MEIO AMBIENTE DO RIO GRANDE DO NORTE.Termo de referência. Disponível em: < http://www.idema.rn.gov.br/arquivos/Licenciamento/Documentos\%20T\%C3\%A9cnicos\% 20por\%20Atividade\%20ou\%20Tipo\%20de\%20Servi\%C3\%A7o/Extra\%C3\%A7\%C3\%A3 o\%20de\%20Combust\%C3\%ADvel\%20F\%C3\%B3ssil/TR\%20RCA\%20Pocos\%20Petrolif eros.pdf $>$. Acesso em: 30 de Jul. 2009.

COTOVICZ JUNIOR, Luiz Carlos. O licenciamento ambiental da produção petrolífera onshore, do rio Grande do Norte: uma análise do descarte da água produzida contida em relatórios de controle ambiental (RCAs). Natal, 2009. 
ECOPLAM. Perfuração de três poços exploratórios de petróleo denominados de 3-PTX-9, 3-PTX-9A e 3-PTX-9B no Município de Governador Dix-Sept Rosado/RN - Bacia Potiguar - Terrestre. 1 CD ROM.

ECOPLAM. Perfuração de Seis Poços de Petróleo No Campo de Porto Carão Município de Carnaubais - RN, 2008. 1 CD ROM.

MACÊDO, Sérgio Luiz. Procedimentos de Licenciamento Ambiental da atividade Petrolífera Onshore. Centro Federal de Educação Tecnológica do Rio Grande do Norte (CEFET-RN). Natal, 2007

MARTINS, Rosiney Araújo. Implementação do programa de monitoramento ambiental na fase de implantação: Estudo de caso no município de Governador Dix Sept Rosado - RN. Natal, 2009.

SÁNCHEZ, Luis Enrique. Avaliação de impactos ambientais: conceitos e métodos. São Paulo: Oficina de Textos, 2006.

SILVA, Valdenildo Pedro da. Métodos e técnicas de pesquisa. Centro Federal de Educação Tecnológica do Rio Grande do Norte (CEFET-RN). Natal, 2007.

OLIVEIRA, Karina Angelica de Souza Lima. Análise do processo de licenciamento ambiental onshore no RN, com enfoque na visão dos técnicos do IDEMA. Natal, 2009.

PEGADO, Erika Araújo da Cunha. Legislação Ambiental. Centro Federal de Educação Tecnológica do Rio Grande do Norte (CEFET-RN). Natal, 2007.

THOMAS, José Eduardo. Fundamentos de engenharia de petróleo. Rio de janeiro: Interciência: PETROBRAS, 2001. 